\title{
ESTUDIO SOBRE LAS DIMENSIONES DEL VALOR DE MARCA PRIORIZADAS POR ESTUDIANTES UNIVERSITARIOS QUE PERTENECEN A LA GENERACIÓN Z. (UNA APLICACIÓN DE ECUACIONES ESTRUCTURALES).
}

Study On Market Value Dimensions Prioritized By University Students Belonging To Z Generation

(An Application Of Structural Equations).

Estudo Sobre as Dimensões do Valor da Marca Priorizada por Estudantes Universitários da Geração Z. (Uma Aplicação de Equações Estruturais).

Received: august $/ 2020$

Accepted: november $/ 2020$

Available online: december/2020

Juan Carlos Poveda Velasco, Magister en Administración y Finanzas, Universidad Mayor Real

Pontificia de San Francisco Xavier de Chuquisaca, Bolivia. E-mail:juancapoveda@gmail.com

Iván Marcelo Poveda Velasco, Doctor en Educación Superior, Universidad Mayor Real y

Pontificia de San Francisco Xavier de Chuquisaca, Bolivia. E-mail: marcelo.poveda@gmail.com

\begin{abstract}
Resumen: La investigación tiene el objetivo de identificar cuáles son las dimensiones del valor de marca que priorizan los estudiantes de la Facultad de Contaduría Pública y Ciencias Financieras que pertenecen a la Generación Z; Es de tipo exploratoria, aplicada, cuantitativa y descriptiva. La generación $Z$ es una población que poco a poco se está incorporando al mundo laboral, representa un $25 \%$ de la población mundial y se convertirá en los próximos años en un mercado potencial de mucha influencia en la economía global. La investigación aporta al conocimiento de las características de esta importante cohorte poblacional, enfocándose en describir cuantitativamente las percepciones en relación al Valor de Marca. El estudio está sustentado en dos modelos de medición del Valor de Marca, propuesto uno por Martín y Brown, y el otro por Lassar, Mittal y Sharma. Se elaboró un cuestionario híbrido en base a los dos modelos, que fue aplicada a una muestra probabilística aleatoria para población finita conocida al 95\% de confianza y 5\% de error de 184 estudiantes de la Facultad de Contaduría Pública y Ciencias Financieras de la Universidad Mayor, Real y Pontificia de San Francisco Xavier de Chuquisaca en Bolivia. Los resultados no son concluyentes, sin embargo, responden a las características psico-sociales descritas por diferentes autores, en relación a este grupo poblacional.
\end{abstract}

Palavras-chave : Valor de Marca, Generación Z, Ecuaciones Estructurales. 


\begin{abstract}
The objective of the research is to identify the dimensions of brand value that are prioritized by the students of the School of Public Accounting and Financial Sciences that belong to Generation Z; It is exploratory, applied, quantitative and descriptive. Generation $\mathrm{Z}$ is a population that is gradually entering the world of work, represents $25 \%$ of the world population and will become in the coming years a potential market of great influence in the global economy. The research contributes to the knowledge of the characteristics of this important population cohort, focusing on quantitatively describing the perceptions in relation to Brand Value. The study is based on two models of Measurement of Brand Value, proposed by Martin and Brown, and the other by Lassar, Mittal and Sharma. A hybrid questionnaire was elaborated based on the two models, which was applied to a random probabilistic sample for a finite population known at 95\% confidence and 5\% error of 184 students of the Faculty of Public Accounting and Financial Sciences of the Universidad Mayor, Real y Pontificia de San Francisco Xavier de Chuquisaca in Bolivia. The results are not conclusive, however, they respond to the psycho-social characteristics described by different authors, in relation to this population group.
\end{abstract}

Keywords: Brand value, Generation Z, Structural Equations.

Resumo: A pesquisa visa identificar quais são as dimensões do valor de marca que são priorizadas pelos alunos da Faculdade de Ciências Contábeis Públicas e Financeiras que pertencem à Geração Z; É exploratória, aplicada, quantitativa e descritiva. A Geração $\mathrm{Z}$ é uma população que está gradativamente ingressando no mundo do trabalho, representando $25 \%$ da população mundial e se tornará um mercado potencial com grande influência na economia global nos próximos anos. A pesquisa contribui para o conhecimento das características deste importante coorte populacional, com foco em descrever quantitativamente as percepções em relação ao Valor da Marca. O estudo é apoiado por dois modelos de medição, um proposto por Martín e Brown e outro por Lassar, Mittal e Sharma. Um questionário híbrido foi desenvolvido com base nos dois modelos, o qual foi aplicado a uma amostra probabilística aleatória para uma população finita conhecida a 95\% de confiança e 5\% de erro de 184 alunos da Faculdade de Contabilidade Pública e Ciências Financeiras da Universidad Mayor, Real y Pontificia de San Francisco Xavier de Chuquisaca na Bolívia. Os resultados não são conclusivos, porém respondem às características psicossociais descritas por diferentes autores em relação a este grupo populacional.

Palavras-chave: Valor da Marca, Geração Z, Equações Estruturais.

\title{
INTRODUCCIÓN
}

Los estudios generacionales tienden a ser de bastante interés para los investigadores en el área del mercadeo, pues es frecuente que las personas que ocupan un mismo espacio temporal tienden a tener características más o menos homogéneas en términos de aspiraciones, pensamientos y metas (Di Lucca, 2013; Ministerio de Sanidad, 2016; Tango Cuellar, Dalence Vidal, \& Gutierrez Condori, 2016). Entre los diferentes estudios relacionados con el Marketing, el conocer el verdadero valor de una marca y las dimensiones que la explican resulta ser un importante activo empresarial, además de ser un problema que en la actualidad sigue vigente para los ejecutivos de empresas (Corona Vazquez, 2012; Poveda Velasco, Orlandini Gonzalez, \& Tolavi Soruco, 2017; Villarejo Ramos, 2002). 
La Generación Z hace referencia a la cohorte de personas nacidas a partir del año 1996 y aunque todavía no existe un acuerdo general sobre las fechas límites de esta generación, se pone como límite el año 2010; esta generación post-milenio se compone actualmente de adolescentes jóvenes entre los 13 y 25 años (Deusto., 2016a; Di Lucca, 2013; Dutra, 2017).

Esta generación que en su gran mayoría se encuentra todavía en instituciones de formación universitaria o preuniversitaria, poco a poco se irán insertando al mercado laboral convirtiéndose en un mercado emergente de gran importancia, algunos autores indican que representa aproximadamente un 25\% de la población mundial (Deusto., 2016a; Di Lucca, 2013; Dutra, 2017; Jiménez, Cornelio, \& Vera, 2017).

La Universidad Mayor, Real y Pontificia de San Francisco Xavier de Chuquisaca es una institución de educación superior con una población estudiantil de aproximadamente 45.000 estudiantes, donde el $85 \%$ de los estudiantes se encuentran en el rango de edad de la cohorte que pertenece a la Generación Z (UMRPSFXCH/Dirección de Tecnologías de Información y Comunicación, 2017).

Son aún escasos los estudios, en el contexto del marketing, relacionados con la Generación Z, con la investigación se pretende hacer un aporte relacionado con el área específica del valor de marca, en ese sentido se plantea el siguiente problema, ¿Cuáles son las dimensiones del valor de marca priorizadas por los estudiantes universitarios que pertenecen a la Generación Z?

En consecuencia, el objetivo de la investigación es, identificar cuáles son las dimensiones del valor de marca que priorizan los estudiantes de la Facultad de Contaduría Pública y Ciencias Financieras que pertenecen a la Generación Z.

\section{REVISIÓN BIBLIOGRÁFICA}

\section{Generación Z}


La generación Z es el una cohorte poblacional nacida entre los años 1996 y 2010, también llamados pos-mileniales; desde su nacimiento están inmersos en las redes sociales, el comercio electrónico, los servicios bajo demanda, y hacen uso de la tecnología como algo natural en sus vidas; están acostumbrados a los medio virtuales para sus interacciones sociales, compras, diversión, actividades académicas y otras funciones cotidianas (Deusto., 2016b; Di Lucca, 2013; Dutra, 2017; Tango Cuellar et al., 2016).

Esta generación es experta en el uso de redes virtuales para localizar información, la Generación $\mathrm{Z}$ usa las herramientas virtuales fluidez, muchos de ellos aprendieron el uso de móviles o tablets antes incluso de leer o escribir (Deusto., 2016a; Di Lucca, 2013; Ministerio de Sanidad, 2016). Algunas aproximaciones indican que el $70 \%$ de esta población usa teléfonos inteligentes, y que algo más del 55\% tienen alguna cuenta en redes sociales; Casi un tercio (29\%) declara pasar más de 10 horas a la semana con su teléfono (Ministerio de Sanidad, 2016).

\begin{abstract}
La sociedad digital está adquiriendo una nueva dimensión desde la llegada de los dispositivos móviles conectados. A partir de 2007, coincidiendo con el nacimiento del iPhone, el auge de los dispositivos conectados ha sido exponencial, impulsando la hiperconectividad de buena parte de la sociedad y provocando usos y hábitos que han configurado una nueva categoría en la reciente historia digital... Para la Generación Z el móvil ya no se entiende como un dispositivo con el que solo se puede hablar por teléfono sino como un medio para estar conectado de forma ubicua. Es el elemento de integración digital más importante entre ellos (Cerezo, 2016, p.102).
\end{abstract}

La generación $\mathrm{Z}$ tiene una perspectiva más tolerante en relación a la concepción del mundo, entienden mejor la diversidad y roles sociales, tienden a ser autosuficientes y bastante creativos. Tienen la aspiración de lograr un empleo que impacte al mundo, son afines a algún tipo de voluntariado y son muy sensibles en relación al impacto que tiene el ser humano sobre el planeta.

De alguna manera están acostumbrados a solucionar sus problemas y necesidades de manera personal, están confiados que el acceso a las redes virtuales les posibilita tener acceso a las herramientas necesarias para construir su propio mundo (Lara \& Ortega, 2016; Magallón Rosa, 2016). En cuanto a sus características en entornos de aprendizaje e influencia, existe coincidencia 
en algunos autores, en que esta generación siente gran atracción por la información visual debido a su familiaridad con dispositivos digitales y su facilidad para llevar a cabo diversas actividades simultáneamente, por ello es previsible que a través de la información recibida por los medios tecnológicos tomen decisiones laborales y comerciales (Deusto., 2016b; Di Lucca, 2013; Lara \& Ortega, 2016; Magallón Rosa, 2016).

\section{Valor de marca}

Valor de marca tiene relación directa con la rentabilidad que genera una marca por su posición de reconocimiento en el mercado. El valor de la marca, desde el marketing, describe el valor de tener una marca bien reconocida, está basada en la idea de que una marca específica puede generar más rentabilidad por la percepción que tienen los usuarios de la misma marca (Corona Vazquez, 2012; Villarejo Ramos, 2002).

Los estudios para dimensionar el valor de marca que un grupo poblacional percibe son diversos y tienen importancia para las empresas; conocer las percepciones y creencias que tienen los consumidores, así como las asociaciones mentales que realizan, resultan ser determinantes en sus decisiones de compra (Lambin, Galluci, \& Sicurello, 2009).

El valor de marca está relacionado con la percepción de los consumidores sobre las propiedades extrínsecas de un bien o servicio, así como la capacidad que tiene de satisfacer las necesidades del consumidor; tener una adecuada lectura de las percepciones del valor que los consumidores dan a una marca resulta en una gran fortaleza de las empresas para definir una adecuada estrategia de marketing (Lambin et al., 2009; Poveda Velasco et al., 2017; Villarejo Ramos, 2002).

Existen diversos modelos para dimensionar el valor de marca, los más frecuentes consideran factores relacionados con aspectos financieros, sociales y motivacionales; Intentar buscar concordancia entre los diferentes modelos existentes, resulta tarea difícil, por la diversidad de criterios, sin embargo, un buen número de autores coinciden que dos modelos tiene mayor 
aceptación, el de Martín y Brown propuesto en 1990 y el de Lassar, Mittal y Sharma propupesto en 1995 (Corona Vazquez, 2012; Villarejo Ramos, 2002).

- Martín y Brown, proponen las siguientes dimensiones para medir el valor de marca: Calidad percibida, Valor percibido, Compromiso, Imagen de marca, y Confianza.

- Lassar, Mittal y Sharma, proponen las siguientes dimensiones: Rendimiento, Imagen social, Valor, Confianza, y Compromiso.

Aunque ambos modelos tienen sus propios ítems explicativos para cada dimensión, existen similitudes en la medición de algunos de ellos, al referirse a una misma cualidad de percepción de valor de marca.

\section{METODOLOGÍA}

La investigación es de tipo exploratoria, aplicada, cuantitativa y descriptiva. Exploratoria porque permite ampliar el conocimiento sobre un grupo poblacional específico y sus características, sin excluir la posibilidad de nuevas investigaciones; Es aplicada porque permite entender mejor la percepción de valor de marca y sus dimensiones de medición, a partir de un grupo específico generacional; es cuantitativa al medir las dimensiones que influyen sobre el valor de marca; es descriptiva al caracterizar el valor de marca a partir de las dimensiones y sus ítems que las componen (Morles, 2002; Morone, 2015; Robles, 2018).

La población de referencia son los estudiantes de la Facultad de Contaduría Pública y Ciencias Financieras de la Universidad Mayor, Real y Pontificia de San Francisco Xavier de Chuquisaca, Bolivia, que se encuentran en el rango de edad 17 a 25 años, perteneciente a la denominada Generación Z (Deusto., 2016a, 2016b; Dutra, 2017; UMRPSFXCH/Dirección de Tecnologías de Información y Comunicación, 2017). 
Se realizó una muestra probabilística aleatoria para población finita conocida al $95 \%$ de confianza y 5\% de error, dando un total de 184 estudiantes a ser encuestados, considerando la proporción por género se aplicaron 117 a mujeres y 67 a varones.

La aplicación del cuestionario fue realizada en el segundo semestre del año 2019. Se realizó una adaptación de las dimensiones e ítems propuestos en el modelo de Martín y Brown y el modelo de Lassar, Mittal y Sharma, para desarrollar la encuesta. La encuesta queda definida con treinta y ocho (38) preguntas cerradas de alternativas de respuestas delimitadas, de tipo dicotómicas, de selección múltiple y escala de Lickert con cinco opciones: "totalmente en Desacuerdo", "en Desacuerdo", "No corresponde", "de Acuerdo", "totalmente de Acuerdo". La aplicación de la encuesta fue a través de la aplicación de Formularios del Google Drive.

\section{RESULTADOS, ANÁLISIS Y DISCUSIÓN}

\section{Modelo Estructural a ser Analizado}

El modelo estructural propuesto para su análisis tiene seis variables latentes, de las cuales cinco son independientes - Calidad percibida y Rendimiento; Valor Percibido; Compromiso; Imagen de Marca y Social; Confianza - y una dependiente - Valor de Marca -; El diseño de resumen gráfico fue realizado con apoyo del software SmartPLS V.3.

Figura 1. Modelo estructural del Valor de Marca 


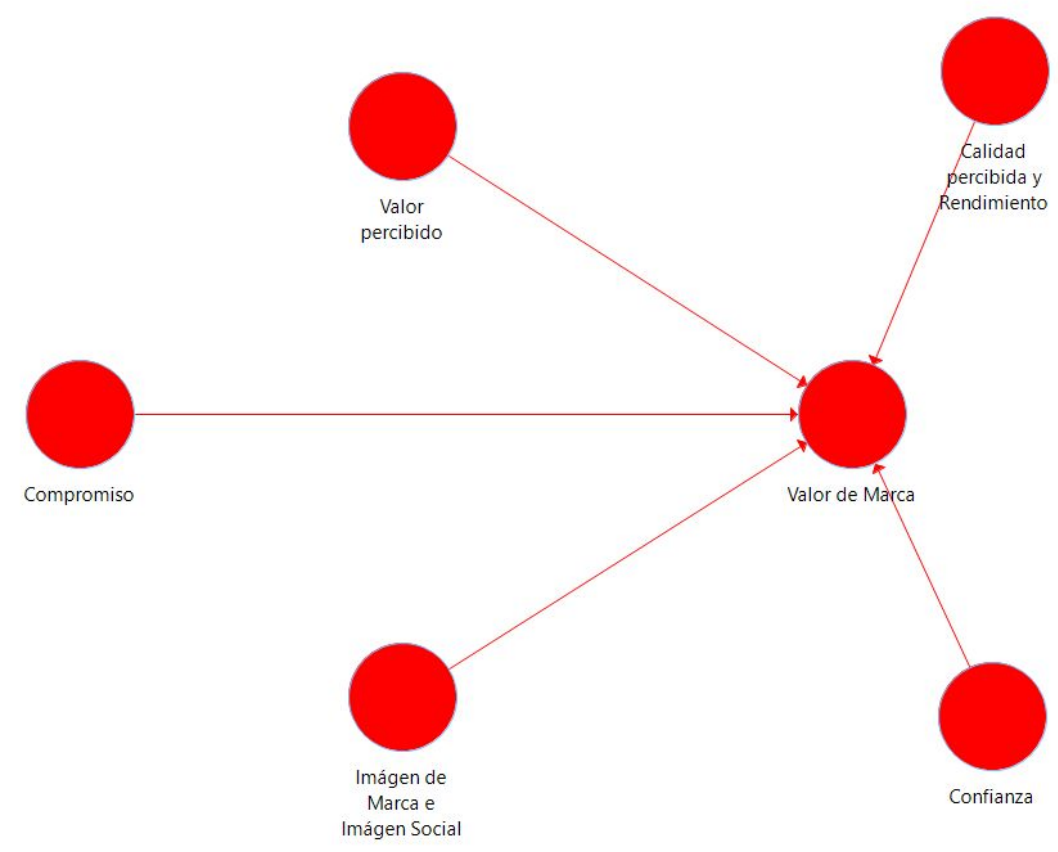

Fuente: Adaptación de Martín y Brown (1990) y de Lassar, Mittal y Sharma (1995)

Gráfico con apoyo del SmartPLS V.3

\section{Validez y Confiabilidad del Modelo}

Para verificar la validez y confiabilidad del modelo se recurre al software SmartPLS V.3. En la Figura 2 se muestra el modelo después del análisis realizado, donde los círculos representan las variables latentes, los rectángulos representan los indicadores aplicados y las flechas el sentido de influencia de las variables. 


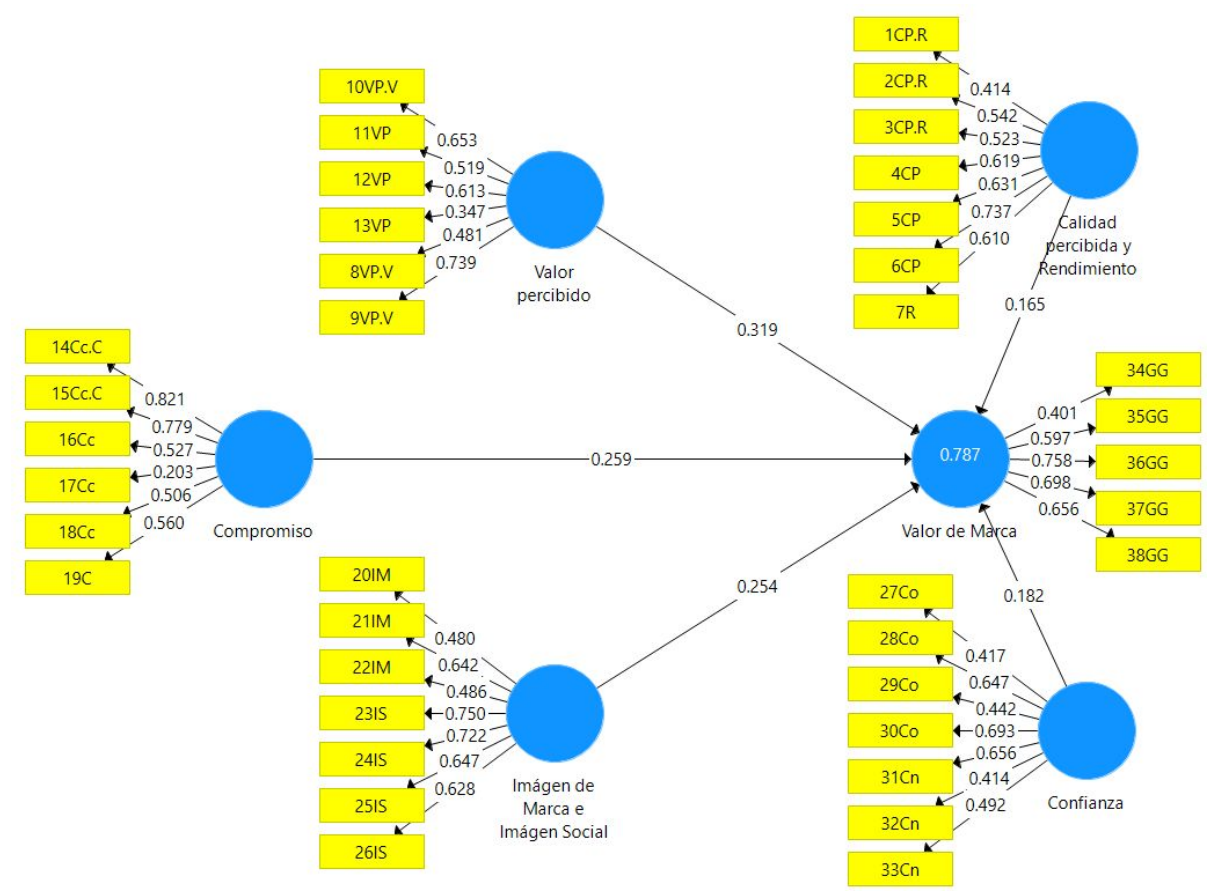

Figura 2. Modelo estructural de Valor de Compra. Confiabilidad interna inicial

Fuente: Encuesta aplicada 2018. Gráfico con apoyo del SmartPLS V.3

Los números en las flechas que enlazan las variables a los indicadores son los valores de confiabilidad, expresados por correlación simple, los indicadores con índices $<0.7$ deben ser depurados por considerarse poco confiables. Los números en las flechas que enlazan las variables entre sí explican el nivel de influencia de una sobre la otra y se denomina coeficiente path o valores Beta $(\beta$ ) los valores $\beta \geq 0.2$ son significativos (Ayaviri, Saucedo, Poveda Velasco, \& Mariano, 2017; Calvo-Porral, Martínez-Fernández, \& Juanatey-Boga, 2013; Leyva Cordero \& Olague, 2014; Ramirez-Correa \& Mariano, 2014).

Realizada la depuración progresiva de los valores menores a 0.7 se obtiene una nueva figura, que se muestra en la Figura 3, donde los valores corresponden a lo exigido por la evaluación de confiabilidad interna siendo todos iguales o mayores a 0.7 . 


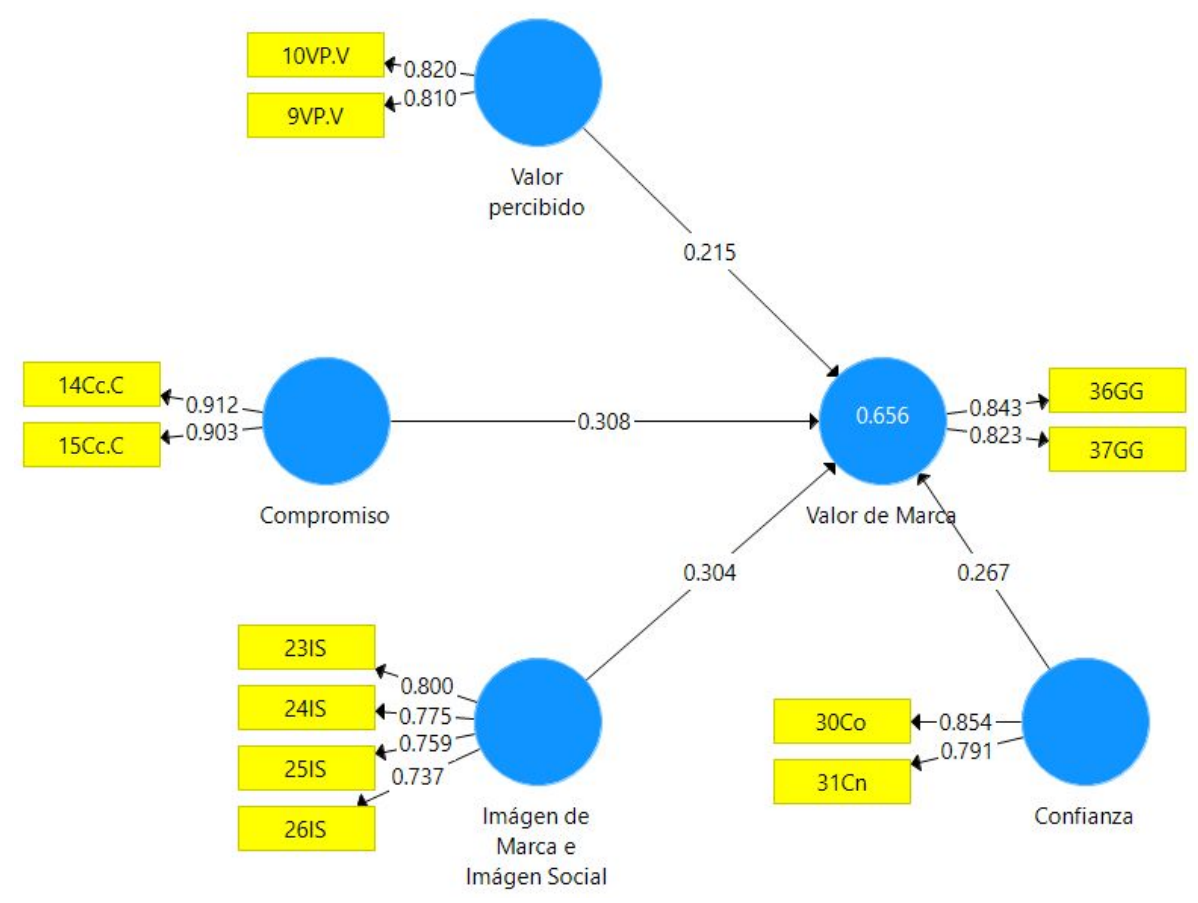

Figura 3. Modelo estructural de Valor de Compra. Confiabilidad final

Fuente: Encuesta aplicada 2018. Gráfico con apoyo del SmartPLS V.3

Según el análisis de fiabilidad por el criterio de Fornell y Lacker propuesto en 1981, el análisis factorial confirmatorio del modelo es analizado a partir de la existencia de una validez discriminante entre dos variables latentes si la varianza compartida entre pares de constructos es menor que la varianza extraída para cada constructo individual, en la Tabla 1 se muestran los resultados de este criterio (Ayaviri et al., 2017; Leyva Cordero \& Olague, 2014; Martínez García \& Martínez Caro, 2009; Ramirez-Correa \& Mariano, 2014).

Tabla 1. Fiabilidad del modelo estructural de Valor de Compra. Criterio de Fornell y Lacker

\begin{tabular}{|c|c|c|c|c|c|}
\hline Dimensión & Compromiso & Confianza & $\begin{array}{c}\text { Imágen de } \\
\text { Marca e } \\
\text { Imágen Social }\end{array}$ & $\begin{array}{c}\text { Valor de } \\
\text { Marca }\end{array}$ & $\begin{array}{c}\text { Valor } \\
\text { percibido }\end{array}$ \\
\hline Compromiso & 0,907 & & & & \\
\hline Confianza & 0,273 & 0,823 & & & \\
\hline Imagen de Marca e Imagen Social & 0,608 & 0,422 & 0,768 & & \\
\hline
\end{tabular}




\begin{tabular}{|l|lll|l|l|}
\hline Valor de Marca & 0,620 & 0,539 & 0,700 & 0,833 \\
\hline Valor percibido & 0,254 & 0,281 & 0,452 & 0,505 & 0,815 \\
\hline
\end{tabular}

Fuente: Encuesta aplicada a estudiantes 2018. Tabla con apoyo del SmartPLS V.3

Para verificar la fiabilidad interna del modelo se procede a la evaluación de confiabilidad a partir del Alfa de Cronbach, Fiabilidad compuesta y la Varianza extraída media. El test con el coeficiente de fiabilidad de constructo muestra que los indicadores agrupados se correlacionan conjuntamente con sus variables recomendando que el valor para el alfa de Cronbach y la Fiabilidad compuesta debe ser igual o mayor a 0.7 ; en relación al test de validez convergente que permite identificar las consistencias internas del modelo, se recomienda que la la Varianza Extraída Media (AVE), debe tener un valor mínimo de 0.5 , significando que más del $50 \%$ de la varianza del constructo se encuentra explicada por sus propios indicadores estando altamente correlacionados (Ayaviri et al., 2017; Calvo-Porral et al., 2013; Leyva Cordero \& Olague, 2014; Ramirez-Correa \& Mariano, 2014).

Tabla 2. Fiabilidad del modelo estructural de Valor de Compra. Fiabilidad y validez del constructo

\begin{tabular}{|c|c|c|c|}
\hline Dimensión & $\begin{array}{c}\text { Alfa de Cronbach } \\
\qquad \mathbf{0 . 7}\end{array}$ & $\begin{array}{c}\text { Fiabilidad compuesta } \\
>\mathbf{0 . 7}\end{array}$ & $\begin{array}{c}\text { Varianza extraída } \\
\text { media (AVE) } \\
>0.5\end{array}$ \\
\hline Compromiso & 0,786 & 0,903 & 0,823 \\
\hline Confianza & 0,719 & 0,808 & 0,678 \\
\hline Imagen de Marca e Imagen Social & 0,769 & 0,852 & 0,590 \\
\hline Valor de Marca & 0,760 & 0,819 & 0,694 \\
\hline Valor percibido & 0,766 & 0,799 & 0,665 \\
\hline
\end{tabular}

Fuente: Encuesta aplicada a estudiantes 2018. Tabla con apoyo del SmartPLS V.3 
La Tabla 2 muestra los valores obtenidos para los indicadores de fiabilidad y validez del modelo corregido, en el que se puede verificar que todos los factores tienen valores mayores a los parámetros requeridos para cada uno de los indicadores.

\section{Valoración del modelo estructural}

Concluido el análisis de validez y confiabilidad del modelo estructural se procede al análisis de Boothstrapping, que permite verificar la estabilidad de las estimaciones proporcionadas por el análisis PLS, con ello se estima la significancia del modelo a partir de los valores de T student que deben ser iguales o mayores a 1.96 (Ayaviri et al., 2017; Calvo-Porral et al., 2013; Ramirez-Correa \& Mariano, 2014). En la Figura 4 los coeficiente $\beta$ representados por las flechas corresponden al valor de T student, donde se observa que todos los valores $\beta$ son mayores a 1.96.

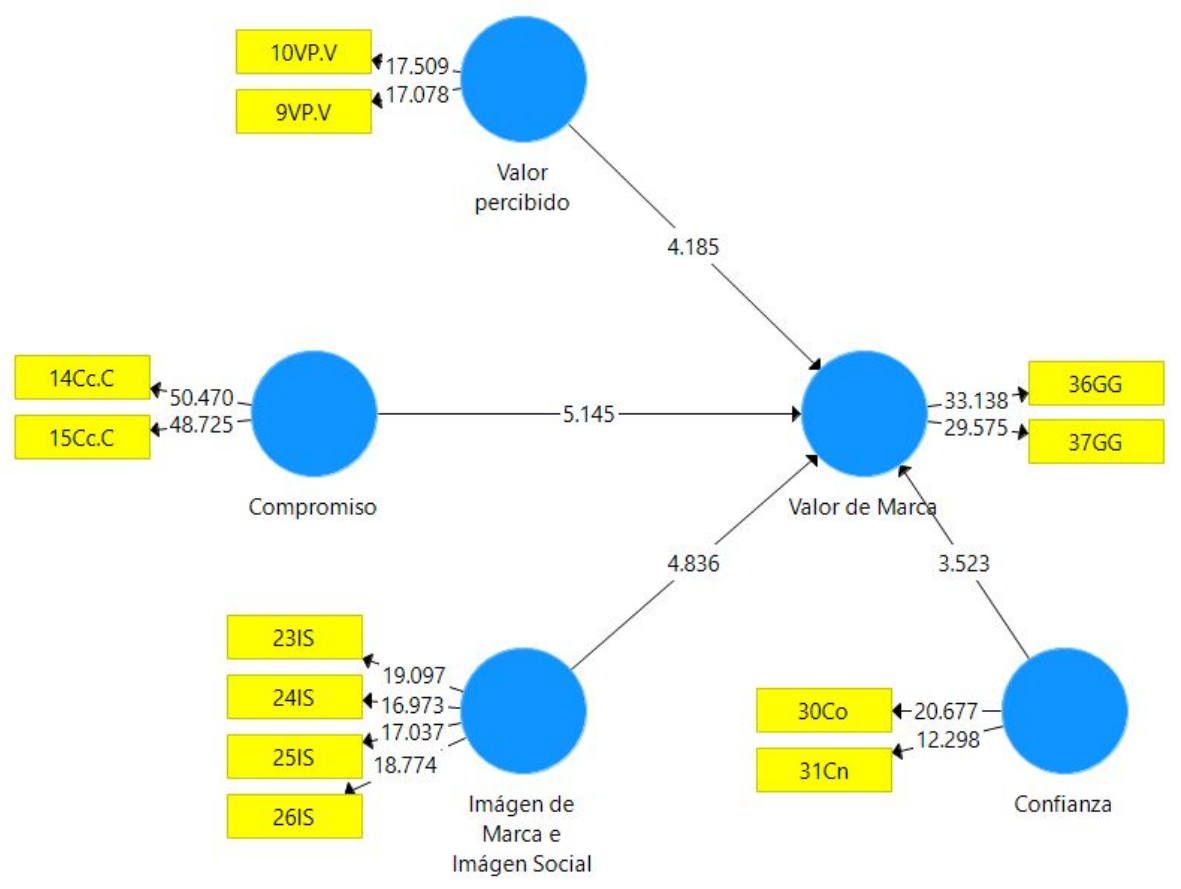

Figura 4. Modelo estructural Valor de Marca. Boothstrapping valor T student $\geq 1.96$

Fuente: Encuesta aplicada 2018. Gráfico con apoyo del SmartPLS V.3 
Se procede al análisis el valor de la variable explicada que se mide con el coeficiente de correlación de Pearson (R2) indicando la relación lineal entre la variable dependiente y las variables independientes (Ayaviri et al., 2017; Leyva Cordero \& Olague, 2014; Ramirez-Correa \& Mariano, 2014), en el modelo estructural analizado el valor es de 0.656 , que significa que la variable Valor de Marca es explicada en el 65.6\% por las variables independientes, ver la Figura 3.

Finalmente, se verifica si el factor de inflación de colinealidad (VIF) es menor a 4.0; los autores indican que existe multicolinealidad cuando el valor del VIF $>0.4$ lo que aumentaría el error estándar (Calvo-Porral et al., 2013; Ramirez-Correa \& Mariano, 2014).

Tabla 3. Modelo estructural Valor de Marca. Evaluación del factor de inflación de colinealidad

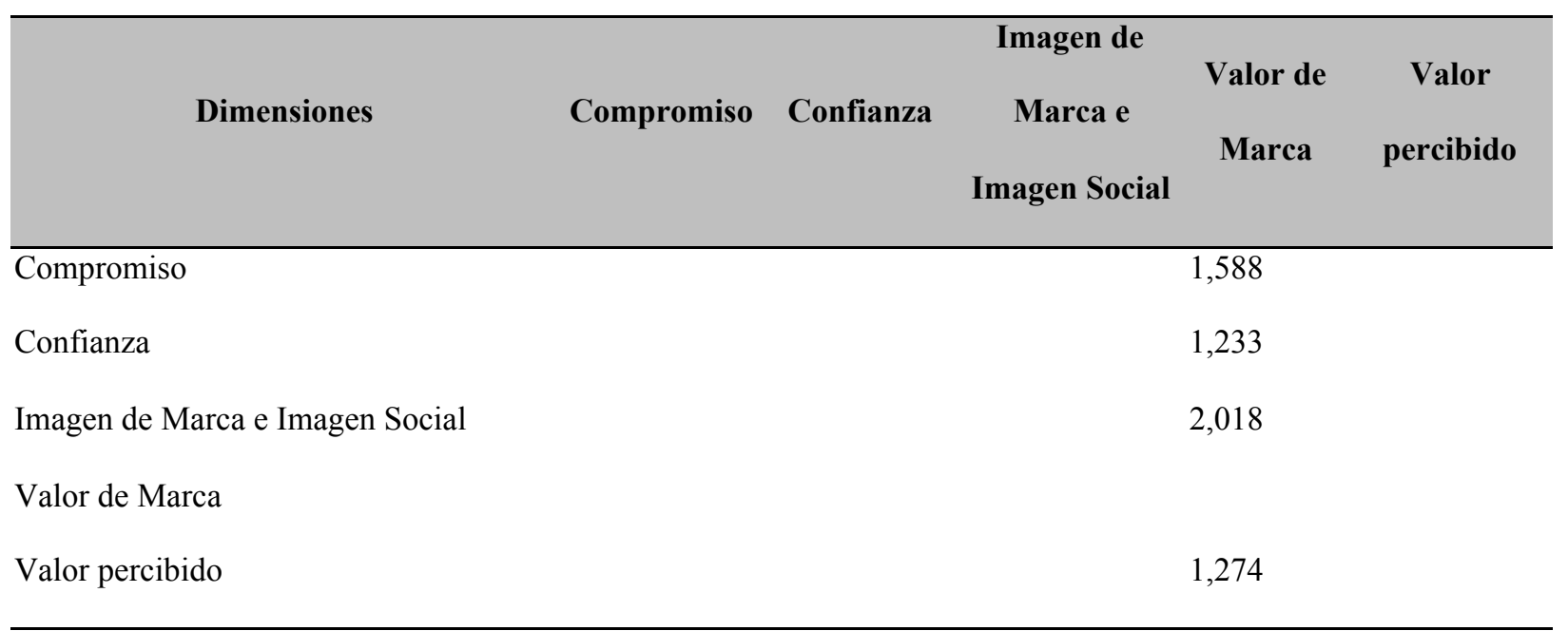

Fuente: Encuesta aplicada a estudiantes 2018. Tabla con apoyo del SmartPLS V.3

En la Tabla 3, se identifica que los valores de colinealidad son menores a 4.0.

\section{CONCLUSIÓN}

El objetivo de la investigación fue identificar cuáles son las dimensiones del valor de marca que priorizan los estudiantes de la Facultad de Contaduría Pública y Ciencias Financieras que pertenecen a la Generación Z; a partir de la evaluación cuantitativa a partir del análisis de un modelo estructural híbrido entre los modelos de Martín y Brown propuesto en 1990 y el de Lassar, Mittal y Sharma propupesto en 1995, aplicado a una muestra de estudiantes universitarios, entre 17 
a 25 años, de la Facultad de Contaduría Pública y Ciencias Financieras de la Universidad Mayor,

Real y Pontificia de San Francisco Xavier de Chuquisaca, las variables estudiadas explican en un 65.6\% las percepciones en relación al Valor de Marca.

Las dimensiones estudiadas, variables independientes, fueron las siguientes:

- Compromiso

- Confianza

- $\quad$ Imagen de Marca e Imagen Social

- Valor percibido

- $\quad$ Calidad percibida - Rendimiento.

De estas cinco dimensiones, la referida a Calidad percibida - Rendimiento, fue depurada del modelo, por tener una fiabilidad inferior a 0.7 y un coeficiente $\beta$ menor a 0.2 (Ayaviri et al., 2017; Calvo-Porral et al., 2013; Leyva Cordero \& Olague, 2014; Ramirez-Correa \& Mariano, 2014). Lo que significa que desde la percepción de los estudiantes universitarios resulta poco relevante en cuanto al Valor de Marca.

Los valores $\beta$ de las otras dimensiones al ser mayores a 0.2 , muestran el nivel de influencia sobre la percepción Valor de Marca, a partir de la Figura 3 en la Tabla 4 se muestran los valores $\beta$ de cada dimensión en relación a la variable Valor de Marca.

Tabla 4. Modelo estructural Valor de Marca. Coeficientes $\beta$

\begin{tabular}{lcc}
\multicolumn{1}{c}{ Dimensión } & Valor de $\boldsymbol{\beta}$ & \multicolumn{1}{c}{ Ítems } \\
\hline Compromiso & 0.380 & 14Cc.C Para comprar el producto debo sentir un cierto cariño por él. \\
& & 15Cc.C Con el tiempo, para comprar un producto deberé sentir un especial \\
& & afecto por el producto \\
Imagen de Marca / & 23IS El producto que compre debe adecuarse a mi personalidad \\
Imagen Social & & 24IS Debo sentirme orgulloso de tener ese producto en específico \\
& & 25IS El producto deberá tener una gran aceptación entre mis amigos
\end{tabular}


26IS El estilo del producto corresponderá a mi propia personalidad

Confianza

Valor percibido
0.267

0.215
30Co Para comprar un producto debe considerarse "serio" o "formal"

31Cn Deberé confiar en la empresa y las personas que fabrican ese producto, antes de comprar

9VP.V El producto me aportará un valor superior al precio pagado por él 10VP.V El producto debe ser una auténtica ganga

Fuente: Encuesta aplicada a estudiantes 2018. Elaboración propia

La dimensión "Compromiso", es la que mayor influencia tiene sobre la percepción de Valor de Marca, con un valor $\beta=0.380$; al verificar los ítems que explican esta dimensión, se observa que la variable compromiso hacia la marca sólo es estimada mediante percepciones de los consumidores en la medida que se generen relaciones de afecto con la marca con el transcurrir del tiempo.

En relación a la "Imagen de Marca / Imagen Social”, la dimensión se encuentra en segundo lugar de importancia; la percepción de Valor de Marca está muy relacionada con las características individuales del consumidor en estrecha relación con la influencia que ejerzan sus amigos; existen rasgos de individualidad pero en el contexto social donde se desenvuelve.

Respecto a la "Confianza", la dimensión ocupa el tercer lugar en importancia, respecto a la valoración de marca; los ítems que explican la dimensión están relacionadas con la formalidad que exprese la marca, así como el compromiso que sea percibido de la empresa respecto con la sociedad.

La dimensión "Valor Percibido", ocupa el último lugar en relación a la percepción de valor de marca; la valoración será mayor en la medida que el producto tenga un precio relativo menor al mismo tiempo que aporten una satisfacción mayor en relación a productos similares.

Los resultados tienen una gran aproximación a las características psico-sociales de la Generación Z descrita por diferentes autores, (Cerezo, 2016; Deusto., 2016b; Di Lucca, 2013; Dutra, 2017; Lara \& Ortega, 2016; Magallón Rosa, 2016; Ministerio de Sanidad, 2016), son autosuficientes aunque susceptibles de ser influidos por sus contextos sociales - personales o 
virtuales -; son bastante prácticos y pragmáticos, no desarrollan un apego inmediato y solo lo harán en función a sus expectativas personales; son ahorradores y previsores en relación a su economía presente y futura, buscan un alto rendimiento con el menor esfuerzo.

La investigación no es concluyente, abre la posibilidad a réplicas en otras facultades y otras universidades, tanto en Bolivia como fuera de ella. Es recomendable continuar con la profundización del estudio de un grupo poblacional que está en desarrollo y altamente globalizado.

\section{REFERÊNCIAS}

Ayaviri, A., Saucedo, H., Poveda Velasco, J. C., \& Mariano, A. M. (2017). Uso de Ecuaciones Estructurales en la Intención Emprendedora de los Estudiantes Universitarios Bolivianos : Caso de la Universidad San Francisco Xavier de Chuquisaca Use of Structural Equations in the Entrepreneurial Intention of Bolivian University Stude. Congresso Internacional de Administração: Conhecimento a Alavanca Do Desenvolvimento, 1-12.

Calvo-Porral, C., Martínez-Fernández, V.-A., \& Juanatey-Boga, O. (2013). Análisis de dos modelos de ecuaciones estructurales alternativos para medir la intención de compra. Revista Investigación Operacional, 34(3), 230-243. Retrieved from http://rev-inv-ope.univ-paris1.fr/files/34313/34313-05.pdf

Cerezo, P. (2016). La Generación Z y la información. Revista de Estudios de Juventud, (114), 95-109.

Corona Vazquez, G. (2012). Comportamiento del Consumidor.

Deusto., A. / D. B. S. U. de. (2016a). Generación Z. El último salto generacional. Resumen Ejecutivo.

Deusto., A. / D. B. S. U. de. (2016b). Generación Z - II Fase. Resumen Ejecutivo.

Di Lucca, S. (2013). El comportamiento actual de la Generación Z en tanto futura generación que ingresará al mundo académico.

Dutra, M. F. (2017). Generación Z : entre las nuevas formas de organización del trabajo y la convivencia generacional.

Jiménez, A. L. L., Cornelio, R. R., \& Vera, Y. J. (2017). Gestion Del Conocimiento En Universidades Públicas Mexicanas. European Scientific Journal, 13(1), 54-70. https://doi.org/10.19044/esj.2017.v13n1p54

Lambin, J., Galluci, C., \& Sicurello, C. (2009). Dirección de Marketing, Gestión Estratégica y Operativa del Mercado. Mcgraw-Hill / Interamericana de México. 2da Edición.

Lara, I., \& Ortega, I. (2016). Los consumidores de la Generación Z impulsan la transformación 
digital de las empresas. Revista de Estudios de Juventud, (114), 71-82.

Leyva Cordero, O., \& Olague, J. T. (2014). Modelo de Ecuaciones Estructurales por el Método de Mínimos Cuadrados Parciales (Partial Least Squares-PLS). TIRANT HUMANIDADES MÉXICO Métodos y Técnicas Cualitativas y Cuantitativas Aplicables a la Investigación en Ciencias Sociales.

Magallón Rosa, R. (2016). El ADN de la Generación Z. Entre la economía colaborativa y la economía disruptiva. Revista de Estudios de Juventud, (114), 29-44.

Martínez García, J. A., \& Martínez Caro, L. (2009). Los test estadísticos y la evaluación de escalas; el caso de la validez discriminante. Investigaciones Europeas de Direccion y Economia de La Empresa, 15(2), 15-24. https://doi.org/10.1016/S1135-2523(12)60086-0

Ministerio de Sanidad, S. S. e I.-E. (2016). Los auténticos nativos digitales: ¿estamos preparados para la Generación Z? Revista de Estudios de Juventud (Vol. 114).

Morles, V. (2002). Sobre la metodología como ciencia y el método científico: un espacio polémico. Revista de Pedagogía, 23(66), 16.

Morone, G. (2015). Métodos y técnicas de la investigación científica.

Poveda Velasco, J. C., Orlandini Gonzalez, I. E., \& Tolavi Soruco, I. B. (2017, September). Responsabilidad Social Universitaria e imagen de marca: percepción desde los estudiantes universitarios. Congreso Internacional de Administración, 12.

Ramirez-Correa, P. E., \& Mariano, A. M. (2014). Propuesta Metodológica para aplicar modelos de ecuaciones estructurales con PLS: El caso del uso de bases de datos científicas en estudiantes universitarios. Revista ADMpg Gestão Estratégica, 7(2), 133-139. Retrieved from https://www.researchgate.net/publication/276410281\%0APropuesta

Robles, F. (2018). Tipos de Investigación. Retrieved from https://www.lifeder.com/tipos-investigacion-cientifica/\%0ALos

Tango Cuellar, A. R., Dalence Vidal, J. P., \& Gutierrez Condori, M. (2016). Características del consumidor de la Generación Z en la ciudad de Sucre. Revista Digiltal Investigación y Negocios, 9(14), 93-101.

UMRPSFXCH/Dirección de Tecnologías de Información y Comunicación. (2017). Universidad Mayor, Real y Pontificia de San Francisco Xavier de Chuquisaca. Estadísticas sobre evolución de la matrícula regular. Gestiones 2013 - 2017. DTIC.

Villarejo Ramos, A. F. (2002). Módelos multidimensionales para la medición del valor de marca. Investigaciones Europeas de Dirección y Economía de La Empresa, 8(3), 13-44. 


\section{Anexo \\ Cuestionario aplicado}

\section{Sexo}

Rango de Edad

Ingreso promedio de mi familia

$\begin{array}{clllr} & \text { Entre Bs. } & \text { Entre Bs. } & \text { Entre Bs. } & \\ 20.001 & 15.001 \mathrm{a} & 10.001 \mathrm{a} & 5.001 \mathrm{a} & \text { Menor a } \\ \text { o más } & 20.000 & 15.000 & 10.000 & \text { Bs. 5.000 }\end{array}$

Menor a

\begin{tabular}{|c|c|c|c|c|c|}
\hline & $\begin{array}{c}\text { Tot } \\
\text { alm } \\
\text { ent } \\
\mathrm{e} \\
\mathrm{de} \\
\mathrm{acu} \\
\text { erd } \\
\mathrm{o}\end{array}$ & $\begin{array}{c}\text { De } \\
\text { acuer } \\
\text { do }\end{array}$ & $\begin{array}{l}\text { Ni de } \\
\text { acuerd } \\
\text { o ni } \\
\text { en } \\
\text { desac } \\
\text { uerdo }\end{array}$ & $\begin{array}{c}\text { En } \\
\text { desac } \\
\text { uerdo }\end{array}$ & $\begin{array}{c}\text { Tota } \\
\text { lmen } \\
\text { te en } \\
\text { desa } \\
\text { cuer } \\
\text { do }\end{array}$ \\
\hline \multicolumn{6}{|l|}{ 1CP.R Elegiré el producto que funcionará muy bien } \\
\hline \multicolumn{6}{|l|}{$\begin{array}{l}\text { 2CP.R El producto no debe ocasionar imprevistos durante } \\
\text { su uso }\end{array}$} \\
\hline \multicolumn{6}{|l|}{$\begin{array}{l}\text { 3CP.R El rendimiento del producto será superior a otros } \\
\text { similares }\end{array}$} \\
\hline \multicolumn{6}{|l|}{ 4CP El producto deberá tener una larga duración } \\
\hline \multicolumn{6}{|l|}{$\begin{array}{l}5 \mathrm{CP} \text { La calidad del producto debe ser superior a otros } \\
\text { productos }\end{array}$} \\
\hline \multicolumn{6}{|l|}{ 6CP El producto deberá ser de alta calidad } \\
\hline \multicolumn{6}{|l|}{ 7R El producto deberá ser fácil de usar } \\
\hline \multicolumn{6}{|l|}{ 8VP.V El producto deberá tener un precio adecuado } \\
\hline \multicolumn{6}{|l|}{$\begin{array}{l}\text { 9VP.V El producto me aportará un valor superior al precio } \\
\text { pagado por él }\end{array}$} \\
\hline \multicolumn{6}{|l|}{ 10VP.V El producto debe ser una auténtica ganga } \\
\hline \multicolumn{6}{|l|}{$\begin{array}{l}11 \text { VP Compraré el producto por que estoy preocupado por } \\
\text { la relación calidad - precio }\end{array}$} \\
\hline \multicolumn{6}{|l|}{$\begin{array}{l}\text { 12VP El producto comparado con otros del mismo precio } \\
\text { debe ser el mejor }\end{array}$} \\
\hline \multicolumn{6}{|l|}{$\begin{array}{l}\text { 13VP Atendiendo a las prestaciones del producto, } \\
\text { consideraré que su precio sea justo }\end{array}$} \\
\hline \multicolumn{6}{|l|}{$\begin{array}{l}\text { 14Cc.C Para comprar el producto debo sentir un cierto } \\
\text { cariño por él }\end{array}$} \\
\hline \multicolumn{6}{|l|}{$\begin{array}{l}\text { 15Cc.C Con el tiempo, para comprar un producto deberé } \\
\text { sentir un especial afecto por el producto }\end{array}$} \\
\hline \multicolumn{6}{|l|}{$\begin{array}{l}\text { 16Cc Aunque el precio de otros productos sea inferior } \\
\text { probablemente compraré el mismo producto }\end{array}$} \\
\hline \multicolumn{6}{|l|}{$\begin{array}{l}\text { 17Cc Para adquirir un producto debo encontrar diferencia } \\
\text { entre el producto y otros similares }\end{array}$} \\
\hline \multicolumn{6}{|l|}{$\begin{array}{l}\text { 18Cc Adquiriré el mismo producto siempre que tenga } \\
\text { necesidad de comprarlo }\end{array}$} \\
\hline \multicolumn{6}{|l|}{$\begin{array}{l}\text { 19C Elegiré el producto sobre el que yo tenga opinión } \\
\text { positiva }\end{array}$} \\
\hline \multicolumn{6}{|l|}{ 20IM En general elegiré el producto que sea mejor que otros } \\
\hline \multicolumn{6}{|l|}{ 21IM Elegiré el producto que tenga buena reputación } \\
\hline \multicolumn{6}{|l|}{$\begin{array}{l}\text { 22IM Deberán existir más opiniones positivas que negativas } \\
\text { para que elija un producto }\end{array}$} \\
\hline \multicolumn{6}{|l|}{$\begin{array}{l}\text { 23IS El producto que compre debe adecuarse a mi } \\
\text { personalidad }\end{array}$} \\
\hline \multicolumn{6}{|l|}{$\begin{array}{l}\text { 24IS Debo sentirme orgulloso de tener ese producto en } \\
\text { específico }\end{array}$} \\
\hline $\begin{array}{l}\text { 25IS El producto deberá tener una gran aceptación entre mis } \\
\text { amigos }\end{array}$ & & & & & \\
\hline
\end{tabular}




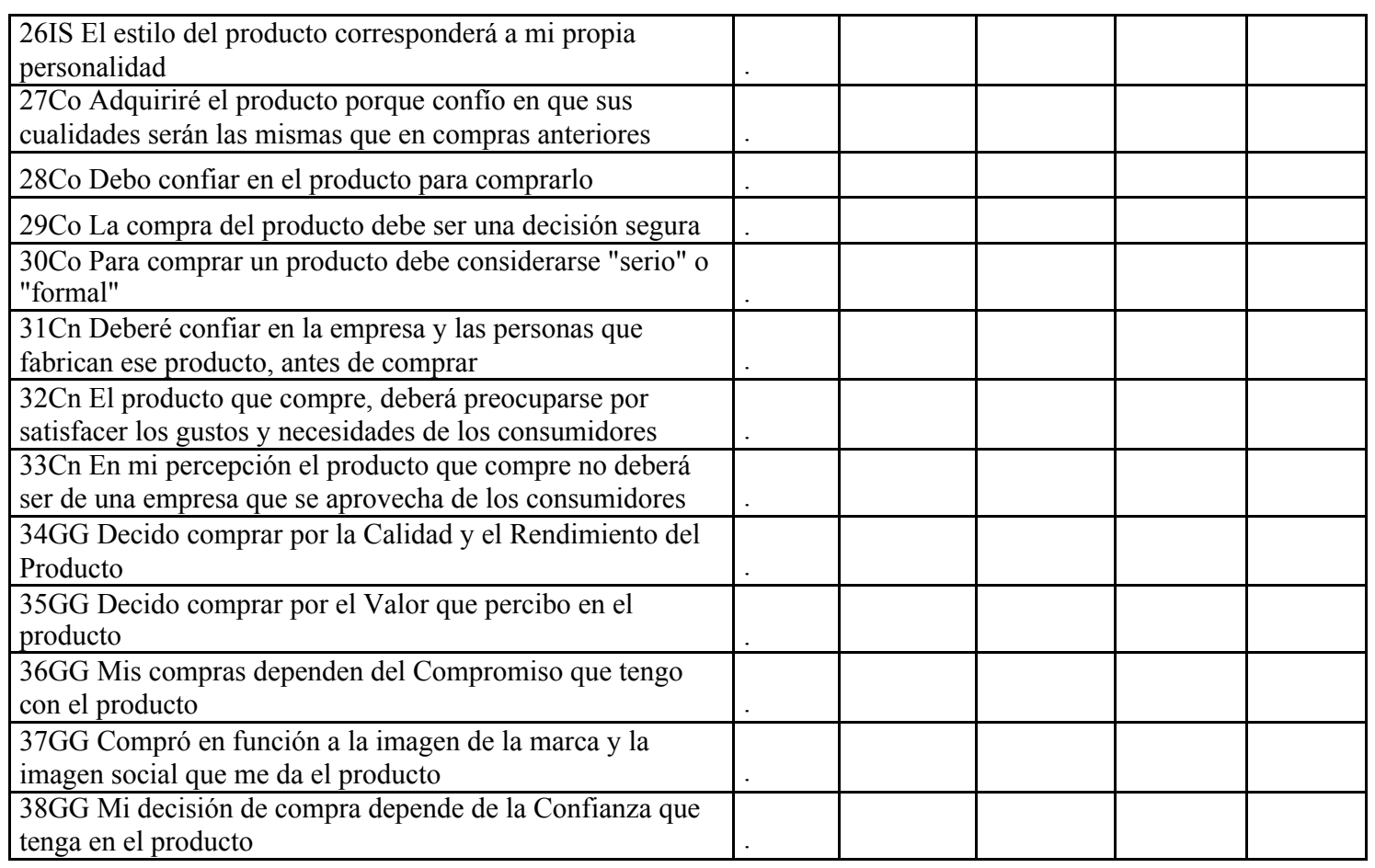

